\title{
The Complex Quantum-State of Black-Hole and Thermostatistics.
}

\author{
Dr. Narayan Kumar Bhadra \\ L.S.S.S. High School (H.S); Gobardanga, North 24 Parganas, West Bengal, India.
}

\begin{abstract}
In this paper the quantum aspects are described in-detail with the help of a new type of energy source called latent energy group SU(6) of the super unified theory of $S U(11)$. The thermodynamics of general self gravitating systems created by the energy group $S U(6)$ and some related topics such as complex space-time (i.e. Pseudo-Space-Time) are also briefly discussed. Black-Hole.

The thermodynamic connection is based on Hawking celebrated application of quantum theory to

We know that macroscopic systems have definite and precise energies, subject to definite conservation principle. In this paper the generalized theory of black-hole is examined in-detail. We study the quantum state of black-hole. We consider our universe begins through the symmetry breaking of the super unified theory with gauss group $S U(11)[\supset S U(6) \times S U(5) \times U(1)]$ from 7-dimensionsl space-time. The subgroup $S U(6)$ has been interpreted as a new type of energy source other than $S U(5)[S U(5) \supset S U(3) \times S U(2) \times U(1)$, where $S U(3)$, the strong energy group, $S U(2)$ the weak-energy group and $U(1)$, the electro-dynamics]. The energy group $S U(6)$ are responsible for the situation of the so-called black-hole and U(1), also for the creation of Schwarzschild mass ' $M$ ' and angular momentum ' $J$ ', as $U(1)$ are responsible for the creation of electric charge ' $e$ ' where $M$ $\& J$ - depends on ' $e$ ' as shown in this paper]. We consider the line element proposed by the quantum-vacuum Kantowski-Sachs universe without cosmological constant. We also consider the scale factor $b(=i R)$ of the metric to be imaginary and the other $a(=R)$ is real, of the Kantowski-Sachs quantum-state with complex spacetime. Solving the metric tensors we get the energy tensors $T_{0}^{0}=T_{1}^{1}$ and $T_{2}^{2}=T_{3}^{3}$ in the $10(=4+6)$ dimensional space-time under the exchange $R_{I} \leftrightarrow R$. We think from 10-dimensional to 7-dimensional stage, the situation of primordial black-hole formed by the energy group $S U(11)$. The fact is obviously defined within 10dimensional to7-dimensional flat universe and then the black-hole formed within 7-dimensional to 4dimensional closed universe through the symmetry breaking of the energy group $S U(11)$, the negative pressure by $T_{2}^{2}=T_{3}^{3}=-p$ and $T_{0}^{0}$ is define the matter energy density and $T_{1}^{1}$ is define the latent energy density. At the early stage, when $T_{0}^{0}=T_{1}^{1}=\epsilon$. The universe then begins its journey through the phase transition system.
\end{abstract}

\section{Introduction:}

The region inside the horizon, once the star has shrunk away to nothing, is empty (i.e. in another phase) and from the exterior universe, black and inaccessible (according to our instrument). It is therefore called a Black-Hole. Astronomers widely believe that black-hole will form as the natural end state of the evolution of massive stars, but so far there is no direct observational evidence of their existence.

The most general known solutions to Einstein's field equations of general relativity which contains black-holes are the so-called Kerr-Newman family, which describe an asymmetric, matter-free space-time representing a black-hole which rotates and carries an electric charge. These solutions from a three-parameter set, labeled by the total mass energy $\mathrm{M}$, the angular momentum $\mathrm{J}=|\mathrm{J}|$ and the electric charge ' $\mathrm{e}$ '.

The change of a larger group $\mathrm{SU}(11)$ of symmetries to the subgroup $\mathrm{SU}(6) \times \mathrm{SU}(5) \times \mathrm{U}(1)$ is spontaneous by the re-distribution of energy particles. The above subgroup which contains the $\mathrm{U}(1)$ group, there inevitably arises particles (whose annihilation formed charge particles) that have the characteristics of a magnetic mono-pole. Typically, the mass of a mono-pole (in energy units) may be $\sim 10^{19} \mathrm{Gev}$ (Plank energy). Monopoles are highly stable particles and once created they are not destructible. And so they would survive as relics to the present epoch. Hence charge 'e', of the black-hole formed by U(1) and survive as back-ground radiation from the black-hole. The explanation of another two energy groups SU(5) and SU(6) of the SUT energy group $\mathrm{SU}(11)$, we begin with the analogy of ferro-magnetism and crucial role of the Curie-temperature ( $770^{\circ} \mathrm{C}$ for iron). Above this temperature a bar of iron shows no magnetism in an external field. This is because its elementary nuclear magnets are randomly aligned with no resultant magnetization. Energetically, this is the lowest state for the bar and it chooses to remain in that state as the most stable one. Bellow the Curie temperature the state of lowest energy changes to that in which all the nuclei are aligned along the bar, which develops polarity at its ends. There are two states of the same lowest energy possible, depending on which (north or south) of the two poles falls at a given end. The ultimate choice of one state apparently breaks symmetry although theoretically and inherently the symmetry is always there. In the early universe something similar happens to the super unified theory $\mathrm{SU}(11)$ and then $\mathrm{SU}(5)$. Above like a critical temperature $\mathrm{Tc}$, the 
vacuum state, the state of lowest energy, is none other than the potential $\varphi=0$. Below Tc, the state of lowest energy of the thermo-statistical particles are changed. It now corresponds to a situation when $\varphi$ has non-zero values. Corresponding to states of the same lowest energy, let us suppose that there exist alternative values $\varphi_{i}$ (i $=1,2,3, \ldots$ ) which now acquire that status of vacuum. There are basic symmetry with respect to all $\varphi_{i}$, but in practice the system may spontaneously acquire one of them. This is again an apparent break-down of symmetry.

The consequences of this for the very early universe are that it is divided into different domains, each with a different value of $\varphi_{i}$. In this way the universe acquires discontinuities along the domain walls. These translate into highly significant discontinuities of matter distribution. The fact that we do not see such discontinuities in actuality (say in the form of large sheets of matter) is hard to explain away. This difficulty is known as the domain wall problem.

The intersection of two domain walls is a linear structure known as 'cosmic-string' such filamentary structure have been invoked in scenarios for galaxy formation.

\section{Super Unified Theory:}

In the theory of Gauss transformation in physics, the special unitary group is used to represent bosonic symmetries. In the theories of symmetry breaking, it is important to find the subgroups of special unitary group. Important subgroups of SU(n) that are important in GUT physics(also in the present dissertation). Super unified theory are,

$$
\text { For } \quad \mathrm{p}>1, \mathrm{n}-\mathrm{p}>1 \text { with } \mathrm{SU}(\mathrm{n}) \supset \mathrm{SU}(\mathrm{p}) \times \mathrm{SU}(\mathrm{n}-\mathrm{p}) \times \mathrm{U}(1) \text {. }
$$

For completeness there are also the orthogonal and sympletic subgroup : $\quad$ SU(n) $\supset O(n)$; $S U(2 n) \supset U S p(2 n)$

Since the rank of $\mathrm{SU}(\mathrm{n})$ is $\mathrm{n}-1$ and $\mathrm{U}(1)$ is 1 , a useful check is that the sum of the ranks of the subgroups is less than or equal to the rank of original group $\mathrm{SU}(\mathrm{n})$ which is a subgroup of various other lie group: $\mathrm{SO}(2 \mathrm{n}) \supset$ SU(n).

From the symmetry breaking of SU(11), we find SU(6) and SU(5) as the subgroups of SU(11), where p (=5) > $1 ; n-p(=11-5=6)>1$, so that $\mathrm{SU}(\mathrm{n}) \supset \mathrm{SU}(\mathrm{p}) \times \mathrm{SU}(\mathrm{n}-\mathrm{p}) \times \mathrm{U}(1)$. i.e. $\mathrm{SU}(11) \supset \mathrm{SU}(5) \times \mathrm{SU}(6) \times \mathrm{U}(1)$. For completeness there are also the orthogonal and sympletic subgroups: $\mathrm{SU}(11) \supset \mathrm{O}(11)$; $\mathrm{SU}(22) \supset \mathrm{US} \mathrm{p}(22)$.

Since the rank of $\mathrm{SU}(11)$ is 10 and $\mathrm{U}(1)$ is 1 , a useful check is that the sum of the ranks of the subgroup $\mathrm{SU}(5)$ and SU(6) is less than or equal to the rank of the original group. Thus, we have from SU(11), the Hermitian matrix $\mathrm{H}$ has 120 arbitrary constants. Which correspond to 120 bosons that now mediate between the different basic entities. Of these we already have 24 from SU(5) and 35 from SU(6) and 1 from U(1).

Thus, $120-(24+35+1)=60$ more bosons are needed to make up the list of 120 . For want of any specific designation, they are referred to simply as the $\mathbf{J}$ bosons. The $\mathbf{J}$ bosons are expected to link the participants of SU(6) with SU(5) i.e. with SU(2), SU(3) and U(1). There are emitted and absorbed $\bar{J}$ particles (anti-J particles). Therefore, in the theory of SU(11), it is possible to change any of 30(thirty) latent energy bosons of SU(6) into any of the 30(thirty) matter energy bosons of SU(5) or vice-versa by the exchange of the Jbosons of SU(11). So at this stage, by the symmetry breaking of SU(11) created an amount of matter energy SU(5) by the latent energy group SU(6), and an angular momentum 'J' \& Schwarzschild 'M' of the black-hole by the energy group $U(1)$. After then $S U(5)$ breaks into $S U(3) \times S U(2)_{L} \times U(1)$.

In the present dissertation there neither any starting point nor any ending point of the wider (measurable in quantum cosmology) universe (having complex space-time). Only there exists an initial and final condition for narrower (measurable classically) universe (i.e. Einstein 4-dimensional space-time) which emerged from wider universe by the process of changing phase, where it was a continuous process.

Again, on the other-hand, the idea that our 4-dimensional universe might have emerged from a higher dimensional space-time is now receiving much attention where the compactification of higher dimensions plays a key role. However, the question arises of how and why this compactification occurs. From string theory we know that the compactification may take place provided that the higher dimensional manifold admits special properties, namely if the geometry of the manifold allows, for example, the existence of a suitable killing vector. However, it is difficult to understand why such manifolds are preferred and whether other possible mechanism for compactification do exist. In cosmology, on the other-hand, different kinds of compactification could be considered. For example, in an approach, called dynamical compactification, the extra-dimensions evolve in time towards very small sizes and the extra dimensional universe reduces to an effective 4-dimensional one. This type of compactification was considered in my previous published paper ['The complex model of the universe' of IOSR-JM, vol.2, 4 (2012), pp-41] with the help of Modern Kaluza-Klein theories. It is then a natural question that how an effective four dimensional universe evolve in time and whether the resulting cosmology is similar to the standard FRW four dimensional universe without extra dimensions. A universe may based on the considerations of dark energy sector. One may start from a fundamental theory including both gravity and standard model of particle physics. In this regard it is interesting to begin with $10(=4+6)$ dimensional space-time, in which case one needs a compactification of 10-dimensional super gravity theory 
where an effective 4-dimensional undergoes acceleration. However, it has been known for sometime that it is difficult to derive such a cosmology and has been considered that there is a no-go theorem that excludes such a possibility, if one takes the internal space to be time-independent and compact without boundary. However, it has recently been shown that one may avoid this no-go theorem by giving up the condition of timeindependence of the internal space, and a solution of the vacuum Einstein equations with compact hyperbolic internal space has been proposed based on this model.

On the other-hand, from cosmological point of view it is not so difficult to find cosmological models in which the 4-dimensional universe undergoes an accelerating expansion and the internal space contracts with time, exhibiting the dynamical compactification. In my paper we consider the internal space as imaginary (pseudo- space, $b=i R$ ) of the Kantowski-Sachs universe, where the matter belongs to another phase by the phase transition system with the help of the latent energy group SU(6).

\section{Intelligence: $\mathrm{SU}(6)$}

In the transformations under the energy group SU(6), the basic fields here are the latent energy field and we have

$$
\mathrm{U}=\exp (-\mathrm{iH})
$$

Where ' $\mathrm{H}$ ' is a $6 \times 6$ Hermitian matrix of zero-trace. The matrix $\mathrm{H}$ now has 35 independent components. In the weak interaction $\mathrm{SU}(2)$, we have, $\mathrm{H}$ has $2 \times 2$ Hermitian matrix of zero-trace and the most general form of such matrix is

$$
H=\left(\begin{array}{cc}
a & b+i c \\
b-i c & -a
\end{array}\right)=a\left(\begin{array}{cc}
1 & 0 \\
0 & -1
\end{array}\right)+b\left(\begin{array}{ll}
0 & 1 \\
1 & 0
\end{array}\right)+c\left(\begin{array}{cc}
0 & i \\
-i & 0
\end{array}\right)
$$

Thus, like above, we have 35 matrix charges $\mathrm{I}_{1}, \mathrm{I}_{2}, \mathrm{I}_{3}, \ldots \ldots \ldots \mathrm{I}_{35}$ out of which five matrices are diagonal. Corresponding to this, we have 35 bosons. For want of any specific designation, they are referred to simply as $\mathbf{J}_{\mathrm{k}}$. There were no change takes place for exchanging the bosons namely $\mathrm{J}_{\mathrm{k} 3}, \mathrm{~J}_{\mathrm{k} 8}, \mathrm{~J}_{\mathrm{k} 15}, \mathrm{~J}_{\mathrm{k} 24}, \mathrm{~J}_{\mathrm{k} 35}$, corresponding to the said five diagonal matrices. We expect the participating interactions of the bosons $\mathrm{J}_{\mathrm{k}}$ to have comparable strength. The $J_{k}$ bosons are expected to generate a latent force. This force is believed to be potentially so large that the exotic matter fluid are expected to transfer into the ordinary matter field constituting a black-hole at the centre region.

\section{Excess production of $\mathbf{J}_{\mathbf{k}}$ bosons in the very early universe:}

Let us denote the mass of the $\mathrm{J}$-bosons by $\mathrm{m}_{\mathrm{J}}$, and its coupling strength by $\alpha_{\mathrm{J}}$. The coupling strength depending on what type of particle $J$ is, let us denote $b y \Gamma_{C}{ }^{\prime}$ the rate of collisions that do not conserve the number of $\mathrm{J}_{\mathrm{k}}$ bosons, i.e. collisions in which the $\mathrm{J}$-boson is involved. Denote the characteristic decay rate of the $\mathrm{J}$-boson by $\Gamma_{\mathrm{J}}$, we thus have three time scales to play with: $\Gamma_{\mathrm{J}}^{-1}, \Gamma_{\mathrm{e}^{\prime}}^{-1}$ and $\mathrm{H}_{\mathrm{I}}^{-1}$.

At the earliest epochs, with constant temperature $>10^{19} \mathrm{GeV}$, the latent energy was the strongest force between the various constituents of the universe. Other interactions were unimportant under the hypothesis of asymptotic freedom. As the universe continued to changing phase and its constant temperature dropped there is a phase when gravity as well as latent force become weaker while the other interactions still remained unimportant. Thus for $\mathrm{T} \leq 10^{19} \mathrm{GeV}$, the particles remained essentially free for some time.

During this phase it becomes necessary to examine the nature of distribution, functions are as follows. Assuming ideal gas approximation and thermodynamic equilibrium, it is then possible to write down the distribution functions of any given species of particles. Let us use the symbol $L$ to denote typical species $(\mathrm{L}=1$, $2, \ldots)$. Thus $\mathrm{n}_{\mathrm{L}}(\mathrm{P}) \mathrm{dp}$ denotes the number density of species in the momentum range $(\mathrm{P}, \mathrm{P}+\mathrm{dP})$, where

$$
n_{L}(P)=\frac{g_{L}}{2 \pi^{2} \hbar^{3}} P^{2}\left[\exp \frac{E_{L}(P)-\mu_{L}}{k T}-1\right]^{-1}
$$

Where $\mathrm{T}=$ the temperature of the distribution, $\mathrm{g}_{\mathrm{L}}=$ the number of spin states of the species, $\mathrm{k}=$ the Boltzmann constant and $E_{L}^{2}=c^{\prime 2} P^{2}+m_{L}^{2} c^{\prime 4}$ is the energy corresponding to rest mass $m_{L}$ of a typical particle. The quantity $\mu_{\mathrm{L}}$ is the chemical potential of the species $\mathrm{L}$. We set $\mu_{\mathrm{L}}=0, \mathrm{~g}_{\mathrm{L}}=1, \mathrm{~m}_{\mathrm{L}}=0$, for $\mathrm{J}_{\mathrm{k}}$ bosons. Since particles and antiparticles annihilate in pairs and produce $\mathrm{J}_{\mathrm{k}}$ bosons their chemical potentials are equal and opposite. Again we saw that for $\mathrm{T} \leq \mathrm{T}_{\mathrm{J}}$, the distribution function cannot preserve its form under changing phase. Thus it may get distorted from its equilibrium form. Now of the various species in the very early universe, the 
$\mathrm{J}$-bosons are probably the most massive. Thus, provided they have a high enough value $\mathrm{T}_{\mathrm{J}}$, there is a chance that the $\mathrm{J}$ bosons will first dropout of equilibrium. For this to happen, however, it is also necessary that they have not all decayed by then. The collision rate

$\Gamma_{\mathrm{c}^{\prime}} \approx \alpha_{\mathrm{J}}<<\Gamma_{\mathrm{J}}$ A comparison of the three rats shows that $\Gamma_{\mathrm{c}^{\prime}}<\Gamma_{\mathrm{J}}<\mathrm{H}_{\mathrm{I}}$.

Hence, in this situation, the gravity became so strong that means the amount of equivalent energy is adequate and formed like black-hole, i.e. gravitationally strong region at the centre of the matter distribution within event horizon.

And soon after gravity become weak that means the amount of equivalent energy was distributed, then the changing phase of the universe with the essentially no interaction between the species.

\section{The field equation in complex quantum state:}

The work covered in the Einstein field equations did not tell us the important item of information about the universe is what happened, when the volume of the matter universe squeezed into zero volume and there before. To find the answer to this question it is necessary to do beyond the concept of Einstein universe. We need a new concept with the Einstein's universe to proceed any further, and Einstein's general relativity with complex space-time is one of such theory. We will consider alternative approaches to cosmology but for the present is Kantowski-Sachs universe. We have the line element to start with:

$$
\mathrm{ds}^{2}=-\mathrm{N}^{2}(\mathrm{t}) \mathrm{dt}^{2}+\mathrm{a}^{2}(\mathrm{t}) \mathrm{dr}^{2}+\mathrm{b}^{2}(\mathrm{t})\left(\mathrm{d} \theta^{2}+\operatorname{Sin}^{2} \theta \mathrm{d} \phi^{2}\right)
$$

The only nontrivial Einstein equations of the above metric

$$
\begin{aligned}
& T_{0}^{0}=-\frac{c^{\prime 4}}{8 \pi G}\left[\frac{2 \dot{a} \dot{b}}{a b N^{2}}+\frac{\dot{b}^{2}}{b^{2} N^{2}}+\frac{1}{b^{2}}\right] \\
& T_{1}^{1}=-\frac{c^{\prime 4}}{8 \pi G}\left[\frac{2 \ddot{b}}{b N^{2}}-\frac{\dot{b} \dot{N}}{b N^{3}}+\frac{\dot{b}^{2}}{b N^{2}}+\frac{1}{b^{2}}\right] \\
& T_{2}^{2}=-\frac{c^{\prime 4}}{8 \pi G}\left[\frac{\ddot{a}}{a N^{2}}+\frac{\ddot{b}}{b N^{2}}+\frac{\dot{a} \dot{b}}{a b N^{2}}-\frac{\dot{a} \dot{N}}{a N^{3}}-\frac{\dot{b} \dot{N}}{b N^{3}}\right]=T_{3}^{3}
\end{aligned}
$$

For, considering $\mathrm{N}=1$, we have

$$
\begin{gathered}
\frac{\dot{b}^{2}}{b^{2}}+\frac{2 \dot{a} \dot{b}}{a b}+\frac{1}{b^{2}}=-\frac{8 \pi G}{c^{14}} T_{0}^{0} \\
\frac{2 \ddot{b}}{b}+\frac{\dot{b}^{2}}{b^{2}}+\frac{1}{b^{2}}=-\frac{8 \pi G}{c^{/ 4}} T_{1}^{1} \\
\frac{\ddot{a}}{a}+\frac{\ddot{b}}{b}+\frac{\dot{a} \dot{b}}{a b}=-\frac{8 \pi G}{c^{14}} T_{2}^{2}=-\frac{8 \pi G}{c^{14}} T_{3}^{3}
\end{gathered}
$$

Where $c$ is the velocity of photon-like particle in vapor stage and $c^{\prime}>c$, the velocity of photon. We next consider $\mathrm{a}=\mathrm{R} \& \mathrm{~b}=i \mathrm{R}_{\mathrm{I}}$, [where $\mathrm{i}=\sqrt{-1}$ ]. Then the equation (7), (8) \& (9) becomes

$$
\begin{array}{r}
\frac{\dot{R}_{1}^{2}}{R_{1}^{2}}+\frac{2 \dot{R}_{1}}{R R_{1}}-\frac{1}{R_{1}^{2}}=-\frac{8 \pi G}{c^{14}} T_{0}^{0} \\
\frac{2 \ddot{R}_{1}}{R_{1}}+\frac{\dot{R}_{1}^{2}}{R_{1}^{2}}-\frac{1}{R_{1}^{2}}=-\frac{8 \pi G}{c^{14}} T_{1}^{1} \\
\frac{\ddot{R}}{R}+\frac{\ddot{R}_{1}}{R_{1}}+\frac{\dot{R}_{1}}{R R_{1}}=-\frac{8 \pi G}{c^{14}} T_{2}^{2}=-\frac{8 \pi G_{1}}{c^{14}} T_{3}^{3}
\end{array}
$$


Before we consider specific forms of $T_{k}^{i}$, it is worth noting that three properties must be satisfied by the energy tensor in the present framework of cosmology. The first is obviously define negative pressure by $T_{2}^{2}=T_{3}^{3}$. The second $T_{0}^{0}$ is define the matter density and the third $T_{1}^{1}$ is define the latent energy density.

If $T_{2}^{2}=T_{3}^{3}=0$, then $\frac{\ddot{R}}{R}+\frac{\ddot{R}_{I}}{R_{I}}+\frac{\dot{R}}{R} \frac{\dot{R}_{I}}{R_{I}}=0$

If $\mathrm{R}_{\mathrm{I}} \leftrightarrow \mathrm{R}$ at 10-dimensional, then, $t=\frac{R^{\frac{3}{2}}-E}{D}$, [where $\mathrm{D}, \mathrm{E}$ are integration constants].

Again, we consider the case, when

$$
\begin{aligned}
& N(t)=R^{3}(t) R_{I}^{D}(t) i^{D} ; \text { then, } T_{0}^{0}=-\frac{c^{\prime 4}}{8 \pi G}\left[\frac{2 \dot{R} \dot{R}_{I}}{R^{7} R_{I}^{2 D+1} i^{2 D}}+\frac{\dot{R}_{I}^{2}}{R^{6} R_{I}^{2 D+2} i^{2 D}}-\frac{1}{R_{I}^{2}}\right] \\
& T_{1}^{1}=-\frac{c^{\prime 4}}{8 \pi G}\left[\frac{\ddot{R}}{R^{7} R_{I}^{2 D} i^{2 D}}+\frac{\ddot{R}_{I}}{R^{6} R_{I}^{2 D+1} i^{2 D}}-\frac{\dot{R} \dot{R}_{I}^{2}}{R^{7} R_{I}^{2 D+1} i^{2 D}}\right] \\
& T_{2}^{2}=T_{3}^{3}=-\frac{c^{\prime 4}}{8 \pi G} \cdot \frac{1}{R^{6} R_{I}^{2 D} i^{2 D}}\left[\frac{\ddot{R}}{R}+\frac{\ddot{R}_{I}}{R_{I}}+\frac{\dot{R} \dot{R}_{I}}{R R_{I}}-\frac{3 \dot{R}^{2}}{R^{2}}-\frac{D \dot{R} \dot{R}_{I}}{R R_{I}}-\frac{3 \dot{R} \dot{R}_{I}}{R R_{I}}-\frac{D \dot{R}_{I}^{2}}{R_{I}^{2}}\right]
\end{aligned}
$$

Case-I, when $D=6$, then

$$
\begin{aligned}
& T_{0}^{0}=-\frac{c^{\prime 4}}{8 \pi G}\left[\frac{2 \dot{R} \dot{R}_{I}}{R^{7} R_{I}^{13}}+\frac{\dot{R}_{I}^{2}}{R^{6} R_{I}^{14}}-\frac{1}{R_{I}^{2}}\right] \\
& T_{1}^{1}=-\frac{c^{\prime 4}}{8 \pi G}\left[\frac{2 \ddot{R}_{I}}{R^{7} R_{I}^{13}}-\frac{6 \dot{R}_{I}^{2}}{R^{6} R_{I}^{13}}-\frac{12 \dot{R}_{I}^{2}}{R^{6} R_{I}^{14}}+\frac{\dot{R}_{I}^{2}}{R^{6} R_{2}^{14}}-\frac{1}{R_{I}^{2}}\right] \\
& T_{2}^{2}=T_{3}^{3}=-\frac{c^{\prime 4}}{8 \pi G} \cdot \frac{1}{R^{6} R_{I}^{12}}\left[\frac{\ddot{R}}{R}+\frac{\ddot{R}_{I}}{R_{I}}+\frac{\dot{R} \dot{R}_{I}}{R R_{I}}-\frac{3 \dot{R}^{2}}{R^{2}}-\frac{D \dot{R} \dot{R}_{I}}{R R_{I}}-\frac{3 \dot{R} \dot{R}_{I}}{R R_{I}}-\frac{D \dot{R}_{I}^{2}}{R_{I}^{2}}\right] \\
& {\left[\because i^{2}=-1 ; i^{12}=1\right]}
\end{aligned}
$$

Now, at $D=6, R_{I} \leftrightarrow R$, i.e., we have, a single scale factor, by putting $R_{I}=R$,

$$
\begin{aligned}
& T_{0}^{0}=-\frac{c^{\prime 4}}{8 \pi G}\left[\frac{3 \dot{R}^{2}}{R^{20}}-\frac{1}{R_{I}^{2}}\right] \\
& T_{1}^{1}=-\frac{c^{\prime 4}}{8 \pi G}\left[\frac{2 \ddot{R}}{R^{19}}-\frac{17 \dot{R}^{2}}{R^{20}}-\frac{1}{R^{2}}\right] \\
& T_{2}^{2}=T_{3}^{3}=-\frac{c^{\prime 4}}{8 \pi G}\left[\frac{2 \ddot{R}}{R^{19}}-\frac{17 \dot{R}^{2}}{R^{20}}\right]
\end{aligned}
$$

Case-II, when $D=3$, and $R_{I}=R$

$$
\begin{aligned}
& T_{0}^{0}=-\frac{c^{\prime 4}}{8 \pi G}\left[\frac{3 \dot{R}^{2}}{R^{14}}-\frac{1}{R^{2}}\right] \\
& T_{1}^{1}=-\frac{c^{\prime 4}}{8 \pi G}\left[\frac{2 \ddot{R}}{R^{13}}-\frac{11 \dot{R}^{2}}{R^{14}}+\frac{1}{R^{2}}\right] \\
& T_{2}^{2}=T_{3}^{3}=\frac{c^{\prime 4}}{8 \pi G}\left[\frac{2 \ddot{R}}{R^{13}}-\frac{11 \dot{R}^{2}}{R^{14}}\right]
\end{aligned}
$$


Case-III, when $D=0$, and $R_{I}=R$, then

$$
T_{0}^{0}=-\frac{c^{\prime 4}}{8 \pi G}\left[\frac{3 \dot{R}^{2}}{R^{8}}-\frac{1}{R^{2}}\right]
$$

$$
\begin{aligned}
& T_{1}^{1}=-\frac{c^{\prime 4}}{8 \pi G}\left[\frac{2 \ddot{R}}{R^{7}}-\frac{5 \dot{R}^{2}}{R^{8}}-\frac{1}{R^{2}}\right] \\
& T_{2}^{2}=T_{3}^{3}=-\frac{c^{\prime 4}}{8 \pi G}\left[\frac{2 \ddot{R}}{R^{7}}-\frac{5 \dot{R}^{2}}{R^{8}}\right]
\end{aligned}
$$

\section{For Isotropic-Universe (Einstein's 4-dimensional Space-time):}

We have from [case III], i.e. , considering $T_{1}^{1}=T_{2}^{2}=T_{3}^{3}$, then we have,

$$
-\frac{c^{\prime}}{8 \pi G}\left[\frac{2 \ddot{R}}{R^{7}}-\frac{5 \dot{R}^{2}}{R^{8}}-\frac{1}{R^{2}}\right]=-\frac{c^{\prime 4}}{8 \pi G}\left[\frac{2 \ddot{R}}{R^{7}}-\frac{5 \dot{R}^{2}}{R^{8}}\right] \text { i.e. } R \rightarrow \infty .
$$

Thus isotropic universe were possible when the space-time goes to infinity.

\section{Cosmology with complex scale factor:}

Consider the metric in which the space-time is assumed to be of Robertson-Walker type having a complex scale factor $R+i R_{I}$, where the scale factor $R$ stands for $(3+1)$ - dimensional space-time and $i R_{I}(=$ b) is that for the internal space with dimension D. Avoiding the imaginary term, the line element can be expressed in the form

$$
d s^{2}=-N^{2}(t) d t^{2}+R^{2}(t) \frac{d x^{i} d x^{j}}{\left(1+\frac{k r^{2}}{4}\right)^{2}}+b^{2}(t) \frac{d y^{a} d y^{b}}{\left(1+k^{\prime} \rho^{2}\right)^{2}}
$$

where $\mathrm{N}(\mathrm{t})$ is the lapse function, $\mathrm{r}^{2}=\mathrm{x}^{\mathrm{i}} \mathrm{x}^{\mathrm{i}}(\mathrm{i}=1,2,3), \rho^{2}=\mathrm{y}^{\mathrm{a}} \mathrm{y}^{\mathrm{a}}(\mathrm{a}=1,2,3, \ldots \ldots, \mathrm{D})$ and $\mathrm{k}, \mathrm{k}^{\prime}=0, \pm 1$, for flat, closed or open type of 4-dimensional universe and D-dimensional space. For simplicity, we assume the internal space to be flat i.e. $\mathrm{k}^{\prime}=0$. The form of energy-momentum tensor is chosen as

$$
\mathrm{T}_{\mathrm{AB}}=\left(-\rho, \mathrm{p}, \mathrm{p}, \mathrm{p}, \mathrm{p}_{\mathrm{D}}, \mathrm{p}_{\mathrm{D}}, \ldots \ldots \ldots \ldots \ldots, \mathrm{p}_{\mathrm{D}}\right)
$$

Now, we examine the case for which the pressure along all the extra-dimensions vanishes, namely, $p_{D}=0$. In doing so, we are motivated by the brane world scenarios where the matter is to be confined to the 4-dimensional universe, i.e. auxiliary hyper-space, so that all components of $\mathrm{T}_{\mathrm{AB}}$ is set to zero but the space-time components and it means no matter escapes through the extra dimensions.

We assume the energy-momemtum tensor $\mathrm{T}_{\mu v}$ to be that of an exotic fluid with the equation of state

$$
\mathrm{p}_{\chi}=\left(\frac{\mathrm{m}}{3}-1\right) \rho_{\chi}
$$

Where $\mathrm{p}_{\chi}$ and $\rho_{\chi}$ are the pressure and density of the fluid, respectively and the parameter $\mathrm{m}$ is restricted to the range

$0 \leq \mathrm{m} \leq 2$, so that there is violation of strong energy condition and the universe experiences accelerated expansion.

The scalar curvature corresponding to the metric (14) has the expression

$$
R=\frac{-6 R R_{1} N \ddot{R}+6 R R_{1} \dot{N} \dot{R}-2 R^{2} \ddot{R}_{1} N+2 R^{2} \ddot{N} R_{1}-2 R_{1} N^{3} k+R_{1} N^{3} k^{2} r^{2}-6 R_{1} N \dot{R}^{2}-6 R \ddot{R R}_{1} N}{R^{2} N^{3} R_{1}}
$$

Now substituting it into the dimensionally extended Einstein-Hilbert action (without higher dimensional cosmological term) including a matter term indicating the above mentioned exotic fluid the effective Lagrangian becomes

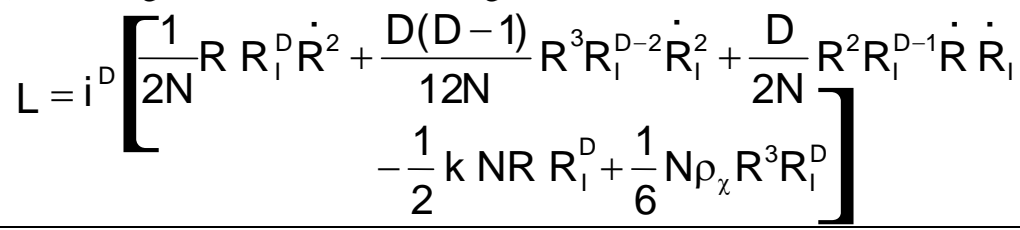


where dot represents the derivative with respect to $\mathrm{t}$. We shall show an equivalence between $(\mathrm{k}=1)$ and $(\mathrm{k}=0)$ universes which are favored by observations. The continuity equation, by using the contracted Bianchi identity in (4+D) dimensions, namely

$$
\nabla_{\mathrm{M}}^{\mathrm{G}}=\nabla_{\mathrm{M}}^{\mathrm{T}^{\mathrm{MN}}}=0
$$

together with the assumption that the matter is confined to $(3+1)$ dimensional space-time gives $\nabla_{\mathrm{i}} \mathrm{T}^{\mathrm{ij}}=0$

$$
\text { i.e. } \quad \dot{\rho}_{\chi} R+3\left(p_{\chi}+\rho_{\chi}\right) \dot{R}=0
$$

Using (9) into the continuity equation (13), the energy density in a closed $(k=1)$ Friedmann-Robertson-Walker universe is

$$
\rho_{\chi}(R)=\rho_{\chi}\left(R_{0}\right)\left(\frac{R_{0}}{R}\right)^{m}
$$

where $R_{o}$ is the value of $R$ at an arbitrary reference time $t_{0}$.

Again, if we believe that the cosmological term plays an important role in vacuum energy density, then we may the cosmological term as

$$
\Lambda \equiv \rho_{\chi}(\mathrm{R})
$$

i.e.

$$
\begin{aligned}
L=i\left[\frac{1}{2 N} R R_{1}^{D} \dot{R}^{2}+\frac{D(D-1)}{1 N}\right. & R^{3} R_{1}^{D-2} \dot{R}_{1}^{2}+\frac{D}{2 N} R^{2} R_{1}^{D-1} \dot{R} \dot{R}_{1} \\
& \left.-\frac{1}{2} N R R_{1}^{D}+\frac{1}{6} N \Lambda R^{3} R_{1}^{D}\right]
\end{aligned}
$$

With

$$
\Lambda(R)=\Lambda\left(R_{0}\right)\left(\frac{R_{0}}{R}\right)^{m}
$$

Taking $\mathrm{m}=2$ and

$$
\Lambda\left(R_{\circ}\right) R_{o}^{2}=3
$$

$$
\Lambda(\mathrm{R})=\frac{3}{\mathrm{R}^{2}}
$$

The lapse function $\mathrm{N}(\mathrm{t})$, is an arbitrary function of time due to the fact that Einstein's general relativity is a reparametrization invariant theory. We therefore, take the gauge

$$
N(t)=R^{3}(t) R_{1}^{D}(t) i^{D}
$$

and then Lagrangian (22) becomes

$$
\mathrm{L}=\frac{1}{2} \frac{\dot{\mathrm{R}}^{2}}{\mathrm{R}^{2}}+\frac{\mathrm{D}(\mathrm{D}-1)}{12} \frac{\dot{\mathrm{R}}_{1}^{2}}{\mathrm{R}_{1}^{2}}+\frac{\mathrm{D}}{2} \frac{\dot{\mathrm{R}}}{\mathrm{R}} \frac{\dot{\mathrm{R}}_{1}}{\mathrm{R}_{1}}
$$

We now define the new variables

$$
\mathrm{X}=\log \mathrm{R}, \quad \mathrm{Y}=\log \mathrm{R}_{\mathrm{I}}
$$

Then the equation (26) becomes

$$
L=\frac{1}{2} \dot{X}^{2}+\frac{D(D-1)}{12} \dot{Y}^{2}+\frac{D}{2} \dot{X} \dot{Y}
$$

The equation of motion are obtained

$$
\ddot{X}+\frac{D}{2} \ddot{Y}=0
$$

$$
\ddot{X}+\frac{D-1}{3} \ddot{Y}=0
$$

Solving equations (29) and (30) we get,

and

$$
\dot{X}=0
$$

$$
\ddot{Y}=0
$$

VIII. Solution of Einstein equations in the complex field:

The most general line element satisfying the Weyl postulate and the cosmological principle is given by

$$
d s^{2}=c^{2} d t^{2}-S^{2}(t)\left[\frac{d r^{2}}{1-k r^{2}}+r^{2}\left(d \theta^{2}+\operatorname{Sin}^{2} \theta d \phi^{2}\right)\right]
$$

where the 3 -space $\mathrm{t}=$ constant are Euclidean for $\mathrm{k}=0$, closed with positive curvature for $\mathrm{k}=+1$ and open with negative curvature for $\mathrm{k}=-1$

We use the above metric to compute the Einstein tensor and thereby formulate the Einstein field equation as 
and

$$
\begin{aligned}
& 2 \frac{S}{S}+\frac{S^{2}+k c^{2}}{S^{2}}=-\frac{8 \pi G}{c^{2}} p \\
& \frac{\dot{S}^{2}+k c^{2}}{S^{2}}=\frac{8 \pi G}{3 C^{2}} \varepsilon
\end{aligned}
$$

Where as usual $\mathrm{p}$ and $\varepsilon$ are the pressure and energy density, respectively.

Now, considering $\mathrm{S}=\mathrm{R}+\mathrm{iR}$,

We have from (34)

$$
2 R \ddot{R}-2 R_{1} \ddot{R}_{1}+\dot{R}^{2}-\dot{R}_{1}^{2}+k c^{2}=-\frac{8 \pi G p}{c^{2}}\left(R^{2}-R_{1}^{2}\right)
$$

$$
=-\frac{8 \pi G p}{c^{2}}\left(R^{2}-R_{1}^{2}\right)-2 i \frac{8 \pi G p}{c^{2}} R R_{1}
$$

and

$$
\begin{aligned}
R \ddot{R}_{1} & +R_{1} \ddot{R}+\dot{R} \dot{R}_{1}=-\frac{8 \pi G p}{c^{2}} R R_{1} \\
& \text { i.e. } \frac{d^{2}}{d^{2}}\left(R t_{1}\right)=\dot{R} \dot{R}_{1}-\frac{8 \pi G p}{c^{2}} R R_{1}
\end{aligned}
$$

Again, from the equation (28) using $\mathrm{S}=\mathrm{R}+\mathrm{iR}_{\mathrm{I}}$ and separating real and imaginary part, we have

$$
\begin{gathered}
\dot{R}^{2}-\dot{R}_{1}^{2}=\frac{8 \pi G \varepsilon}{3 c^{2}}\left(R^{2}-R_{1}^{2}\right)-k c^{2} \\
\dot{R}_{R_{1}}=\frac{8 \pi G \varepsilon}{3 c^{2}} R R_{1}
\end{gathered}
$$

combining equations (31) and (33), we get

$$
\frac{\mathrm{d}^{2}}{\mathrm{dt}^{2}}\left(\mathrm{RR}_{1}\right)=\frac{8 \pi \mathrm{G}}{3 \mathrm{c}^{2}}(\varepsilon-3 p) \mathrm{RR}_{\text {। }}
$$

Also from the Einstein equations (27) \& (28) we have

$$
\begin{gathered}
\frac{d}{d t}\left[S\left(\dot{S}^{2}+k c^{2}\right)\right] \equiv \dot{S}\left[2 S \ddot{S}+\dot{S}^{2}+k c^{2}\right] \\
\text { i.e. } \frac{d}{d}\left(\varepsilon S^{3}\right)+3 p S^{2}=0
\end{gathered}
$$

Now according to second law of thermodynamics the entropy in a given volume $\mathrm{S}^{3}$ remain constant as the volume expands adiabatically. Then $\mathrm{s}=\frac{\varepsilon+\mathrm{p}}{\mathrm{T}}$

is the entropy density and we have

Therefore,

$$
\begin{aligned}
& \frac{d}{d t}\left(S^{3} \cdot s\right)=\frac{d}{d t}\left[\frac{S^{3}}{T}(\varepsilon+p)\right]=0 \\
& \frac{S^{3}}{T}(p+\varepsilon)=\sigma \text { (constant) }
\end{aligned}
$$

Thus using (36), the equation (45) becomes

$$
\frac{\sigma T}{p+\varepsilon}=\left(R+i R_{1}\right)^{3}=\left(R^{3}-3 R R_{1}^{2}\right)+i\left(3 R^{2} R_{1}-R_{1}^{3}\right)
$$

Separating real and imaginary part, we get

So we have

$$
\begin{array}{rr}
\frac{\sigma T}{p+\varepsilon}=R^{3}-3 R R_{I}^{2} & \& R_{I}^{3}-3 R_{I} R^{2}=0 \\
p+\varepsilon=-\frac{\sigma T}{8} \frac{1}{R^{3}} & \text { i.e. } R_{I}= \pm \sqrt{3} R
\end{array}
$$


Using the equation of state (16), $\varepsilon$ is given by $\varepsilon=\frac{3 c^{2}}{3-3 c^{2}-m} \frac{1}{8} \frac{\sigma T}{R^{3}} \quad$ [where $\varepsilon=\rho c^{2}$ ]

Then

$$
\varepsilon-3 p=\frac{3}{8} \frac{c^{2}-m+3}{3-3 c^{2}-m} \frac{\sigma T}{R^{3}}
$$

We have from equations (41) using (46) and (49)

$$
\frac{d}{d t}\left(2 R \frac{d R}{d t}\right)=\frac{A}{R}
$$

[where $A=\frac{\pi G}{c^{2}}\left(\frac{c^{2}-m+3}{3-3 c^{2}-m}\right) \sigma T$, a constant with respect to time ' $t$ '].

Now, integrating the above relation we get

$$
(A R+B)^{1 / 2}(A R-2 B)= \pm \frac{3 A^{2} t}{2}+\frac{3 A C}{2}
$$

where $\mathrm{B}$ and $\mathrm{C}$ are integration constants.

Now, we consider, at $\mathrm{t}=0$, the plank time, $\mathrm{R}(0)=\mathrm{R}_{\mathrm{I}}(0)=\ell_{\mathrm{p}}$.

Then from the above equation we get

$$
2\left(A_{\ell p}+B\right)^{1 / 2}\left(A_{\ell p}-2 B\right)=3 A C
$$

Therefore

$$
t= \pm \frac{2}{3 A^{2}}\left[(A R+B)^{1 / 2}(A R-2 B)-\left(A_{\ell p}+B\right)^{1 / 2}\left(A_{\ell p}-2 B\right)\right]
$$

From, the above equation, we can calculate the exact time, when SU(11) breaks into SU(6), SU(5). That means when the actual vacuum energy breaks into positive energy, negative energy and latent energy.

The solutions for $\mathrm{X}$ and $\mathrm{Y}$ from equations (31) and (32) are

$$
\begin{aligned}
& \mathrm{X}=\mathrm{At}+\gamma, \quad \mathrm{Y}=\mathrm{Bt}+\delta \\
\therefore \quad & \mathrm{R}(\mathrm{t})=\mathrm{A}^{\prime} \mathrm{e}^{\alpha \mathrm{t}} \quad \text { and } \quad \mathrm{R}_{\mathrm{I}}(\mathrm{t})=\mathrm{B}^{\prime} \mathrm{e}^{\beta \mathrm{t}}
\end{aligned}
$$

where the constants $\mathrm{A}, \mathrm{B}, \gamma$ and $\delta$ or $\mathrm{A}^{\prime}, \mathrm{B}^{\prime}, \alpha \& \beta$ should be obtained from the initial conditions.

Consider the size of all spatial dimensions be the same at $\mathrm{t}=0$ and assumed that this size would be the plank size $\ell_{\mathrm{p}}$ in accordance with quantum cosmological considerations.

So we take $\mathrm{R}(0)=\mathrm{R}_{\mathrm{I}}(0)=\ell_{\mathrm{p}} \quad$ so that $\quad \mathrm{A}^{\prime}=\mathrm{B}^{\prime}=\ell_{\mathrm{p}}$.

Thus $\mathrm{R}(\mathrm{t})=\ell_{\mathrm{p}} \mathrm{e}^{\alpha \mathrm{t}} \quad$ and $\quad \mathrm{R}_{\mathrm{I}}(\mathrm{t})=\ell_{\mathrm{p}} \mathrm{e}^{\beta \mathrm{t}}$

It is important to note that the constants $\alpha, \beta$ are not independent and a relation may be obtained between them. This is done by imposing the zero energy condition $\mathrm{H}=0$ which is the well-known result in cosmology due to the existence of arbitrary laps function $\mathrm{N}(\mathrm{t})$ in the theory. The Hamiltonian constraint is obtained through the legender transformation of the Lagrangian (28)

$$
\begin{aligned}
& H=\frac{1}{2} \dot{X}^{2}+\frac{D(D-1)}{12} \dot{Y}^{2}+\frac{D}{2} \dot{X} \dot{Y}=0 \\
& \text { i.e. } H=\frac{1}{2} \alpha^{2}+\frac{D(D-1)}{12} \beta^{2}+\frac{D}{2} \alpha \beta=0
\end{aligned}
$$

This constraint is satisfied only for $\alpha \leq 0, \quad \beta \geq 0$ or $\alpha \geq 0, \beta \leq 0$

To find $\alpha, \beta$, we first obtained the Hubble parameter for $\mathrm{R}(\mathrm{t})$. Again,

Hubble parameter $H_{R}=\frac{\dot{R}}{R}=\alpha$ by which the constant $\alpha$ is fixed.

$$
\mathrm{H}_{\mathrm{I}}=\frac{\dot{\mathrm{R}_{\mathrm{I}}}}{\mathrm{R}_{\mathrm{I}}}=\beta \text { and the }
$$

$$
\therefore \quad \mathrm{R}(\mathrm{t})=\ell_{\mathrm{p}} \mathrm{e}^{\mathrm{H}_{\mathrm{R}} \mathrm{t}}, \quad \mathrm{R}_{\mathrm{I}}(\mathrm{t})=\ell_{\mathrm{p}} \mathrm{e}^{-\mathrm{H}_{\mathrm{R}} \mathrm{t}}
$$

for $\mathrm{D}=1$ also, if $\alpha \neq 0$, then $\alpha=-\beta$ or $\alpha+\beta=0$, or $\quad \dot{\mathrm{X}}+\dot{\mathrm{Y}}=0$, or $\mathrm{X}+\mathrm{Y}=$ constant, or $\log \mathrm{R}+$ $\log R_{I}=$ constant, $\therefore \mathrm{RR}_{\mathrm{I}}=$ constant. Substitute the value of $\mathrm{R}(\mathrm{t})$ from (54) in the equation (52) we get,

$$
t= \pm \frac{2}{3 A^{2}}\left[\left(A_{\ell p} e^{H_{2} t}+B\right)^{1 / 2}\left(A_{\ell p} e^{H_{2} t}-2 B\right)-\left(A_{\ell p}+B\right)^{1 / 2}\left(A_{\ell p}-2 B\right)\right]
$$

Again, for $\mathrm{D} \neq 1$, then either $\alpha=0$ or $\beta=0$

$$
\text { i.e. } \dot{X}=0 \text { or } \dot{Y}=0, \quad \therefore \quad \mathrm{X}=\text { constant, } \quad \mathrm{Y}=\text { constant. }
$$


$\mathrm{R}=\mathrm{R}_{\mathrm{I}}=\ell_{\mathrm{p}}$ (a time independent scale factor)

Therefore, for $\mathrm{D}>1$, we have

$$
\alpha_{ \pm}=\frac{D B}{2}\left[-1 \pm \sqrt{1-\frac{2}{3}\left(1-\frac{1}{D}\right)}\right]
$$

and

$$
\mathrm{H}_{\mathrm{R}^{ \pm}}=\frac{\mathrm{DH}}{2}\left[-1 \pm \sqrt{1-\frac{2}{3}\left(1-\frac{1}{\mathrm{D}}\right)}\right]
$$

So

$$
\begin{aligned}
& \mathrm{R}_{\mathrm{I}}(\mathrm{t})=\ell_{\mathrm{p}} \mathrm{e}^{\mathrm{H}_{\mathrm{I}} \mathrm{t}} \\
& \mathrm{R}_{ \pm}(\mathrm{t})=\ell_{\mathrm{p}} \mathrm{e}^{\frac{\mathrm{DH} \mathrm{H}_{\mathrm{t}} \mathrm{t}}{2}}\left[-1 \pm \sqrt{1-\frac{2}{3}\left(1-\frac{1}{\mathrm{D}}\right)}\right]
\end{aligned}
$$

Substituting (56), (57), (58), (59) in (52), we also get an equation of time t, by solving this, we get, the exact value of the time. It is seen that the time is dependent on the extra-dimensions D. It is easy to show that the Lagrangian (28)

(or the equations of motions) is invariant under the transformation

$$
R \rightarrow R^{-1} ; R_{I} \rightarrow R_{I}^{-1}
$$

Which is consistent with the time reversal $\mathrm{t} \rightarrow-\mathrm{t}$.

For the special case, when $\mathrm{D}=3$ (i.e. $4+3=7$-dimension), both the Lagrangian (28) and the Hamiltonian (53) are invariant under the transformation $\quad R \rightarrow R_{I} ; R_{I} \rightarrow R$.

Therefore, we have a dynamical symmetry between $\mathrm{R}$ and $R_{I}$, namely $R_{I} \leftrightarrow R$. In this case there is no distinction between $R_{I}$ and $R$ to single out one of them as the real scale factor of the universe. Because $k^{\prime}=0$ and according to (26), we assume the $4 \mathrm{D}$-universe with $\mathrm{k}, \Lambda \neq 0$ to be equivalent to the one in which $\mathrm{k}=\Lambda=0$. Therefore both have the same topology $S^{3}$.

So, there is a flatness between 10-dimensional universe to 7-dimensional universe. Hence there is a link with an extremely fine tuning of the universe to the flat $(\mathrm{k}=0)$ model. If this tuning was not there, the universe could either have gone into a collapse $(\mathrm{k}=1)$ or an expansion to infinity $(\mathrm{k}=-1)$ in time scales of the order of $10^{-35} \mathrm{~s}$ that were characteristic of the GUT era.

Now the entropy in a given co-moving volume stays constant in adiabatic expansion. In the entropy was conserved then we would have $\mathrm{RT}=$ constant, where $\mathrm{R}$ is the scale factor and in the black-hole problem $\mathrm{T}=$ $10^{19} \mathrm{GeV}$. However, we found that in the flatness problem this hypothesis led to fine tuning while for the horizon problem it gave an extremely small size of homogeneity. It therefore appears that the trouble of blackhole lies between 10-dimensional to 7-dimensional flat universe and it could be resolved if the adiabatic assumption were violated at this stage. So, we conclude that, within the event horizon from 10-dimensional super-gravity stage, there are mainly two stages, one at the very early stage, were flatness then closed and as well as rotational stages for black-hole. So, in the late time, we have a symmetry breaking of the energy group SU(11) [SUT] of the flat universe at 7-dimensional space-time and gave three fundamental energy groups $\mathrm{SU}(6), \mathrm{SU}(5)$ and $\mathrm{U}(1)$ within the horizon, where U(1) being a charge particle.

\section{Quantum cosmology over complex space:}

Quantum cosmology explain an appropriate quantum mechanical description of the universe, which was introduced and developed by DeWitt. In quantum cosmology the universe, as a whole is treated quantum mechanically and is described by a single wave function, $\psi\left(\mathrm{h}_{\mathrm{ij}}, \phi\right)$ defined on a manifold (super space) of all possible three geometries and all matter field configurations. The wave function $\psi\left(\mathrm{h}_{\mathrm{ij}}, \phi\right)$, has no explicit time dependence due to the fact that there is no real time parameter external to the universe. Therefore, there is no Schrodinger wave equation but the operator version of the Hamiltonian constraint of the Dirac canonical 
quantization procedure, namely vanishing of the variation of the Einstein -Hillbert action S with respect to the arbitrary lapse function $\mathrm{N}$.

Thus, $\quad \mathrm{H}=\frac{\delta \mathrm{S}}{\delta \mathrm{N}}=0$,

which is written as

$$
\hat{\mathrm{H}} \psi\left(\mathrm{h}_{\mathrm{ij}}, \phi\right)=0
$$

This equation is known as the Wheeler-DeWitt (WDW) equation. The goal of quantum cosmology by solving the WDW equation over the complex space $\left(R+i R_{I}\right)$ is to understand the origin and evolution of the universe. In principle, it is very difficult to solve the WDW equation in the super space due to the large number of degrees of freedom. In practice, one has to freeze out of all but a finite number of degree of freedom of the gravitational and matter fields. This procedure is known as quantization in mini-super-space, and will be used in the following.

The mini-super-space in our model is two dimensional with gravitational variables $\mathrm{X}$ and $\mathrm{Y}$. To obtain the Wheller-DeWitt equation, in this mini-super-space, we start with the Lagrangian (28). The conjugate momenta corresponding to $\mathrm{X}$ and $\mathrm{Y}$ are obtained as

$$
\begin{aligned}
P_{x}=\frac{\partial L}{\partial \dot{X}}=\dot{X}+\frac{D}{2} \dot{Y} \\
P_{y}=\frac{\partial L}{\partial \dot{Y}}=\frac{D}{2} \dot{X}+\frac{D(D-1)}{6} \dot{Y}
\end{aligned}
$$

From which we obtain

$$
\begin{aligned}
& \dot{X}=\frac{6}{D+2}\left[P_{x}\left(\frac{1-D}{3}\right)+P_{y}\right] \\
& \dot{Y}=\frac{6}{D(D-1)}\left[P_{y} \frac{2(1-D)}{D+2}-P_{x} \frac{D(1-D)}{D+2}\right]
\end{aligned}
$$

Substituting equations (64) \& (65) into the Hamiltonian constraint (53)

$$
H=(1-D) P_{x}^{2}-\frac{6}{D} P_{y}^{2}+6 P_{x} P_{y}=0
$$

Now, we may use the following quantum mechanical replacements

$$
P_{x} \rightarrow-i \frac{\partial}{\partial x}, \quad P_{y} \rightarrow-i \frac{\partial}{\partial y}
$$

by which Wheeler-DeWitt equations takes the form

$$
\left[(D-1) \frac{\partial^{2}}{\partial X^{2}}+\frac{6}{D} \frac{\partial^{2}}{\partial Y^{2}}-6 \frac{\partial}{\partial X} \frac{\partial}{\partial Y}\right] \psi(X, Y)=0
$$

Where $\psi(\mathrm{X}, \mathrm{Y})$ is the wave function of the universe in the $(\mathrm{X}, \mathrm{Y})$ mini-super-space.

$$
\begin{aligned}
& \text { The general solution is } \psi_{D}(X, Y)=\phi_{1}\left\{D(D-1) Y+3 D X+\sqrt{3 D^{2}+6 D} X\right\} \\
&+ \phi_{2}\left\{D(D-1) Y+3 D X-\sqrt{3 D^{2}+6 D} X\right\} \\
& \text { i.e. } \psi_{D}\left(R, R_{1}\right)=\phi_{1}\left[\log \left\{R^{3 D+\sqrt{3 D^{2}+6 D}} \cdot R_{1}^{D(D-1)}\right\}\right]+\phi_{2}\left[\log \left\{R^{3 D-\sqrt{3 D^{2}+6 D}} \cdot R_{1}^{D(D-1)}\right\}\right]
\end{aligned}
$$

Now for

$$
\psi_{\mathrm{D}}\left(\mathrm{R}, \mathrm{R}_{1}\right) \leftrightarrow \psi_{\mathrm{D}}\left(\mathrm{R}_{1}, \mathrm{R}\right) \text { we have } 3 \mathrm{D} \pm \sqrt{3 \mathrm{D}^{2}+6 \mathrm{D}}=\mathrm{D}(\mathrm{D}-1)
$$

i.e. $\mathrm{D}=1$ or 6 . So if $\mathrm{D}>1$ then we must have $\mathrm{D}=6$. And we take

$$
R_{1}=\ell_{p} e^{H_{1} t} \quad \& \quad R=\ell_{p} e^{H_{R} t}
$$




$$
\text { \& } \quad \mathrm{T}_{2}^{2}=\mathrm{T}_{3}^{3}=\frac{\mathrm{c}^{14}}{8 \pi \mathrm{G}}\left[\frac{\mathrm{e}^{-2 \mathrm{H}_{1} \mathrm{t}}}{\ell_{\mathrm{p}}^{2}}-3 \mathrm{H}_{1}^{2}\right]
$$

Now, it is clear that, when $\boldsymbol{H}_{\boldsymbol{I}}=\boldsymbol{H}_{\boldsymbol{R}}$ then $\boldsymbol{R}_{\boldsymbol{I}} \propto \boldsymbol{R}$. Which is possible for $6+4=10$-dimensional space-time. Then the equations (69) \& (70) are identical

$$
\begin{array}{r}
\mathrm{T}_{0}^{\circ}=\mathrm{T}_{1}^{1}=-\frac{3 \mathrm{c}^{/ 4}}{8 \pi \mathrm{G}}\left[\mathrm{H}_{\mathrm{R}}^{2}-\frac{\mathrm{e}^{-2 \mathrm{H}_{\mathrm{R}} \mathrm{t}}}{3 \ell_{\mathrm{p}}^{2}}\right]=\mathcal{E} \\
\mathrm{T}_{2}^{2}=\mathrm{T}_{3}^{3}=-\frac{3 \mathrm{c}^{/ 4}}{8 \pi \mathrm{G}}\left[\mathrm{H}_{\mathrm{R}}^{2}\right]=-\frac{3 \mathrm{c}^{/ 4}}{8 \pi \mathrm{G}}\left[\mathrm{H}_{1}^{2}\right]=-\mathrm{p} \\
\text { i.e. } \boldsymbol{p}+\boldsymbol{\epsilon}=\frac{c^{\prime 4}}{8 \pi G l_{p}^{2}} \boldsymbol{e}^{-2 H_{R} t}
\end{array}
$$

\section{The quantum state of the black-hole:}

A spherically symmetric black-hole with mass $\mathrm{M}$ and electric charge e is described by the space-time metric

$$
d s^{2}=\left(1-\frac{2 M}{r}+\frac{e^{2}}{r^{2}}\right) d t^{2}-\left(1-\frac{2 M}{r}+\frac{e^{2}}{r^{2}}\right)^{-1} d r^{2}-r^{2}\left(d \theta^{2}+\operatorname{Sin}^{2} \theta d \varphi^{2}\right)
$$

Where $M^{2}>e^{2}$ and $\mathrm{r}$ is a radial co-ordinate chosen to make the surface area of a sphere of radius $\mathrm{r}$ equal to $4 \pi r^{2}$, as in Minkowski-Space.

Comparing the equation (3) with (75), we have

$$
\begin{aligned}
& -N^{2}(t)=1-\frac{2 M}{r}+\frac{e^{2}}{r^{2}} \\
& a^{2}(t)=-\left(1-\frac{2 M}{r}+\frac{e^{2}}{r^{2}}\right)^{-1} \\
& b(t)=i r
\end{aligned}
$$

If we choose, $N(t)=1$, then from (76), we get

$$
\begin{array}{r}
1-\frac{2 M}{r}+\frac{e^{2}}{r^{2}}=-1 \\
\text { i.e. } r=r_{ \pm}=\frac{1}{2} M \pm \frac{1}{2}\left(M^{2}-2 e^{2}\right)^{1 / 2}
\end{array}
$$

Thus from (77) \& (78), we get,

$$
\begin{aligned}
& a^{2}(t)=R^{2}(t)=1 \text { and } b(t)=i R_{I}=i r \\
& \text { i.e. } R_{I}=r_{ \pm}=\frac{1}{2} M \pm \frac{1}{2}\left(M^{2}-2 e^{2}\right)^{1 / 2}
\end{aligned}
$$

When $R=R_{I}$, at 7 - dimensional space-time, then

$$
M^{2}=e^{2}+\frac{e^{4}}{4}+1
$$

Thus, the metric (75) is evidently singular at

$$
r=r_{ \pm} \equiv \frac{1}{2} M \pm \frac{1}{2}\left(M^{2}-2 e^{2}\right)^{1 / 2}
$$

These are not singularities in the geometry itself but in the co-ordinate system $(\mathrm{t}, \mathrm{r})$, similar to these which occur to latitude and longitude on the surface of a sphere at the poles. In fact, the outer surface $r_{+}$corresponds to the event horizon: notice that $r_{+} \rightarrow M$ as $e \rightarrow 0$. This surface has global significance, but locally an internal observer would find nothing unusual about the space-time geometry there. The inner surface $r_{-}$is another type of horizon inside the hole- itself.

\section{Thermodynamics with gravity:}

An astronomers, however, have long had to contend with the conjunction of thermodynamics and gravitation. In the broadest sense, thermodynamics regulates the organization of activity in the universe, and gravity controls the dynamics, at least on the large scale.

One of the central difficulties about the thermodynamics of gravitating systems is the apparent absence of true equilibrium. This problem, which can lead directly to 'peculiar' effects, has long been known to astronomers. Stars are hot, self-gravitating balls of gas inside which the weight of the star is supported by its 
own internal kinetic or zero-point quantum presence. Unlike ordinary laboratory thermodynamic systems, a star is made hotter, not by adding energy, but by removing it. We consider the change takes place by the latent energy group SU(6) into the matter energy group of SU(5) of the super unified group SU(11) and vice-versa. So, initially heat can be found from internal energy of the thermo-statistical particles.

\section{Creation of Black-Holes:}

The key to understanding black-holes, and especially their connection with thermodynamics, is to appreciate the meaning of the so-called event horizon. Consider a very compact and massive star. The strength of gravity [due to the excess production of $\mathrm{J}_{\mathrm{k}}$ bosons of $\mathrm{SU}(6)$ in the very early universe] at its surface can be increased by the decomposition of the energy group SU(11) from another phase by the phase transition system. In the very early universe the production of $\mathrm{J}_{\mathrm{k}}$ bosons are much more and hence more bosons of the matter group SU(5) were increased by changing the energy particles of the group SU(6). According to our assumption, our universe started from the 10-dimensional super-gravity in the large scale and there is a flatness with the compactification of extra-dimension up-to 7-dimensional universe. According to the Einstein's 4-dimensional universe, gravity affects the properties of light, and this is manifestation in the behavior of light rays which leave the surface of the star travelling radially outwards. Because the light has to 'do work' to overcome the surface gravity and escape from the star, its energy, and hence frequency, will be some-what diminished. This famous gravitational red-shift has been measured in light leaving such relatively low gravity objects as the Sun, and the Earth. For more compact and massive objects the red-shift can become enormous. Even on Newtonian grounds it is clear (as pointed out by Peirre Laplace as long as 1798) that when the escape velocity from the surface of a star exceeds that of light, something odd must happen. Accordion to both Newtonian gravity and relativity, this turn of events comes about for a spherical, uncharged star if the radius of the star shrinks below $2 \mathrm{GM} / \mathrm{c}^{2}$, where $\mathrm{M}$ is the mass, $\mathrm{c}$ is the speed of light, and $\mathrm{G}$ is the Newtonian gravitational constants. This size is very small, being of the order of $1 \mathrm{~km}$ for the Sun and $1 \mathrm{~cm}$ for the Earth. A straight-forward calculation shows that as the star approaches the critical radius, the light from its surface becomes red-shifted without limit, so that it can no longer been seen; it is black.

A useful picture of the event horizon can be obtained by imaging spherical wave-fronts of light which are emitted radially outwards from different surfaces $r=$ constants. Those spheres travelling from $r>2 G M / c^{2}$ gradually expands and eventually escape to infinity, but those emitted inside the critical radius actually shrink towards the centre, even though they are emitted in a direction away from the centre. Crudely speaking, the gravity there is so strong that it drags the light back-wards. The event horizon is the spherical surface of light that just escapes to infinity after an infinite duration.

According to relativity, matter and information cannot propagate faster than light, so if light can- not escape from inside the horizon, neither can anything else. Thus, once the star has retreated through this surface it can never return to the outside universe.

The region inside the horizon, once the star has shrunk away to nothing, i.e. in other phase, is empty and from the exterior universe i.e. from the present phase, black and inaccessible. It is therefore called a blackhole. Astronomers widely believe that black holes will form as the natural end state of the evolution of massive stars, but so far there is no direct observational evidence of their existence. The most general known solutions to Einstein's field equations of general relativity which contain black holes are the so-called Kerr-Newman family, which describe an asymmetric, matter-free space-time representing a black-hole which rotates and carries an electric charge. These solutions form a three-parameter set, labeled by the total mass-energy M, the angular momentum $\mathrm{J}=|\mathrm{J}|$ and the electric charge e. For $\mathrm{J}=0$, suggests that flatness occurred from 10-dimensional to 7dimensional universe [ as the equation (28) \& (53) are invariant under the transformation $R_{I} \leftrightarrow R$ ] and then from 7-dimensional to 4-dimensional that black-holes have a very high entropy, and represent in some sense the maximum entropy, equilibrium end state of gravitational collapse.

\section{The Laws of Classical Black-Hole 'Thermodynamics':}

There is a general tendency for self-gravitating systems to grow rather than shrink because gravity always attracts. The behavior between the black-holes and thermodynamic equilibrium systems were noted some time ago (for a review see Carter 1973). In the black-hole case, the inability for light to change from inside the event horizon precludes the escape of any material, so the horizon acts as a sort of asymmetric one-way surface: things can fall in and make the hole bigger but not come out and make it smaller that means changes to another phase. This is reminiscent of the second law of thermodynamics, in which there is an asymmetric tendency for a one-way increase in entropy. The size of the black-hole is analogous to the entropy. The above statement can be explained as, in the theory of SU(11), it is possible to change any of 30-bosons of SU(5) of the matter energy group into any of 30-bosons of SU(6) of the latent energy group and vice-versa by the exchange of J-bosons of SU(11). 
The analogy is almost trivial for a spherical, electrically neutral (Schwarzschild) black-hole. In the more general case of black-holes that possess angular momentum J by SU(6) and electric charge e by $\mathrm{U}(1)$, the size of the black hole depends both on $\mathrm{J}$ and $\mathrm{e}$ in a rather complicated way. If the total surface area of the horizon is used as a measure of size then this is given by the formula (Samrr 1973):

$$
A=4 \pi\left[2 M^{2}-\mathrm{e}^{2}+2 \mathrm{M}^{2}\left(1-\frac{\mathrm{e}^{2}}{\mathrm{M}^{2}}-\frac{\mathrm{J}^{2}}{\mathrm{M}^{4}}\right)^{\frac{1}{2}}\right]
$$

[Where, $M^{2}=e^{2}+\frac{e^{4}}{4}+1$, by the equation (81)]

Where $\mathrm{e}^{2}<\mathrm{M}^{2}$ and $\mathrm{J}^{2}<\mathrm{M}^{4}$ (throughout, units with $\mathrm{G}=\mathrm{c}=1$ will be used) so it is clear at a glance whether a disturbance to the black-hole which changes both e and $\mathrm{J}$, as well as $\mathrm{M}$, will always increase the total area.

An example due to Penrose (1969) concerns a method for extracting mass-energy from a rotating black-hole. The mechanism consists of propelling a small body into the region just outside the event horizon where (due to dragging effect on the space surrounding the black-hole caused by its rotation). Some particle trajectories possess negative energy relative to infinity. Due to the symmetry breaking of SU(11), we get mainly two fragments as $\mathrm{SU}(6)$ and $\mathrm{SU}(5)$, one of which i.e. particles of the energy group $\mathrm{SU}(6)$ is placed on one of these negative energy paths, and this part changes to SU(5), so reduce the total mass $M$ of the hole somewhat and hence the mass-energy of SU(5) thereby released by this sacrificed particles of the latent energy group SU(6) which is ejected to infinity at high speed. During this energy transfer the black-hole's rotation rate is diminished somewhat, so $\mathbf{J}$ also decreases. Thus from equation (83), which shows that when $\mathbf{J}$ decreases, the area A increases, but when $\mathrm{M}$ decreases, the area decreases. The change in $\mathrm{M}$ and $\mathrm{J}$ are therefore in competition, but a careful calculation shows that $\mathrm{J}$ always wins and the area increases.

Actually, if the class of all trajectories is studied, it is found that in general the area increases by an amount corresponding to a considerable fraction of mass energy. However, the efficiency of energy extraction can be improved by approaching closely to a limiting class of trajectories for which the event horizon area remains constant. The limiting case is therefore reversible and corresponds to an isentropic change in thermodynamics. In practice, $100 \%$ efficiency (complete reversibility) would be impossible.

This strong analogy between event horizon area and entropy led to use of the name 'second law' in connection with Hawking's area theorem, which is therefore written as:

$$
\mathrm{dA} \geq 0, \quad \text { [equality corresponding to reversibility] }
$$

There are also analogous of the Zeroth, first and third laws of thermodynamics. From (82), we can obtain;

$$
d M=(8 \pi)^{-1} k d A+\Omega d J+\varphi d e
$$

Where $(8 \pi)^{-1} k \equiv \frac{\partial M}{\partial A}$,etc. which is really just an expression of mass-energy conservation and corresponds to the first law. If A plays the role of entropy then we see from (85), that $\mathrm{k}$ plays the role of temperature $(k d A \sim T d S)$. The interesting thing is that $\mathrm{k}$ can be shown to be constant across the event horizon surface. We thus have an expression of a 'Zeroth' law, analogous to the thermodynamic one of which says that in thermodynamic equilibrium there exists a common temperature parameter for the whole system. The quantity $\mathrm{k}$ is known as the surface gravity of the black-hole. Its significance lies in the fact that it determines the e-folding time which controls the rate at which the collapsing star red-shifts and approaches equilibrium. For a Schwarzschild hole $k=(4 M)^{-1}$ and the constant $8 \pi$ in (85) has been chosen to agree with this. The remaining terms in (85) simply describe the work done (energy extracted) from changes in angular momentum $(\Omega d J)$ and electric charge ( $\varphi \mathrm{de}$ ) and have a very obvious structure: $\Omega$ is the (magnitude of) angular velocity and $\boldsymbol{\phi}$ the electric potential at the event horizon.

Finally, there is the third law. It is straightforward to show that if $\mathrm{J}^{2}$ or $\mathrm{e}^{2}$ become large enough such that:

$$
\frac{J^{2}}{M^{4}}+\frac{e^{2}}{M^{2}}=1 \quad \text { i.e. } \quad J^{2}=\left(\frac{e^{4}}{4}+1\right)\left(e^{2}+\frac{e^{4}}{4}+1\right)
$$

then $\mathrm{k}$ vanishes (although A does not). This corresponds to absolute zero (though with finite entropy). A blackhole with parameters given by (86) is known as an extreme Kerr-Newman black-hole. It is the limiting case of an object which still possesses an event horizon. Should the left-hand side become even infinitesimally greater than one, then the horizon would disappear and we would left with a naked singularity, i.e. the singularity would no longer be invisible inside a black-hole but would be able to influence, and be observed by, the outside universe. This circumstance is considered so undesirable for physics that most physicists believe in the so-called cosmic censorship hypothesis due to Penrose (1969): naked singularities cannot form from gravitational collapse. Cosmic censorship implies the un-attainability of 'absolute zero', $\mathrm{k}=0$ [i.e. condition (86) for an extreme black-hole], so it plays the role of the third law.

\section{The conceptual Foundations of Thermo-statistics:}

Thermo-statistics characterizes the equilibrium state of microscopic system without reference either to the specific forces or to the laws of mechanical response. Instead thermo-statistics characterizes the equilibrium state as the state that maximizes the disorder, a quantity associated with a conceptual framework ("Information 
Theory") outside of conventional physical theory. The question arises as to whether the postulatory basis of thermo-statistics thereby introduces new principles not contained in mechanics, electromagnetism, and the like or whether it borrows principles in unrecognized form from that standard body of physical theory. In either case, what are the implicit principles upon which thermo-statistics rests?

There are, in my view, two essential basis underlying thermo-statistical theory. One rooted in the statistical properties of large complex systems. The second rests in the set of symmetries of the fundamental laws of physics. The statistical feature veils the incoherent complexity of the atomic dynamics, thereby revealing the coherent effects of the underlying physical symmetries. The relevance of the statistical properties of large complex systems is universally accepted and reasonable evident. The essential property is epitomized in the "central limit theorem". Which states (roughly) that the probability density of a variable assumes the "Gaussian" form if the variable is itself the resultant of a large number of independent additive sub-variables. Although one might naively hope that measurements of thermo-dynamic fluctuation amplitudes could yield detailed information as to the atomic structure of a system, the central limit theorem precludes such a possibility. It is this insensitivity to specific structural or mechanical detail that underlies the universality and simplicity of thermo-statistics.

\section{Energy Conservation Principle:}

The development of the principle of conservation of energy has been one of the most significant achievements in the evolution of physics. The first recognition of a conservation principle, by Leibniz in 1693 when Leibniz observed that $\frac{1}{2} m v^{2}+m g h$ is a conserved quantity for a mass particle in the earth's gravitational field. As successively more complex systems were studied it was found that additional terms had to be appended to maintain a conservation principle, but that in each case such an ad- hoc addition was possible. The development of electro-magnetic theory introduced the potential energy of the interaction of electric charges, subsequently to be augmented by the electromagnetic field energy. In 1905 Albert Einstein was inspired to alter the expression for the mechanical kinetic energy, and even to associate energy with stationary mass, in order to maintain the principle of energy conservation. In the 1930 Enrico Fermi postulated the existence of the neutrino solely for the purpose of retaining the energy conservation law in nuclear reaction. And so the process continues, successively accreting additional terms to the abstract concept of energy, which is defined by its conservation law. That conservation law was evolved historically by a long series of successive rediscoveries. It was now based on the assumption of a symmetry of the new type of energy source SU(6) by the time translation $\operatorname{system}(t \rightarrow t+$ constant $)$. It was discussed elaborately in my papers (NK Bhadra; The Complex Model of the Universe; IOSR-JM; 2, 4, pp-41), where we consider that our universe actually started from 10-dimensional space-time instead of 4-dimensional Einstein's universe with the symmetry breaking of the special unitary group SU(11) from 7-dimensional flat space-time, there exists two types of energy sources of the Gaussian energy group SU(6) and SU(5), i.e. it was considered SU(6), a new type of energy source called latent energy group, so the conservation law of energy maintain due to the addition of SU(6) with $\mathrm{SU}(5)$. Hence the space time is actually shifted as $t \rightarrow t_{0}$. So it is conventional therefore to adopt some particular state of a system as a fiducial state, the energy of which [i.e. SU(11)] is arbitrarily taken as zero. The energy of a system in any other state, relative to the energy of the system in the fiducial state, is then called the thermodynamic internal energy of the system in that state and is denoted by the symbol U. So for thermodynamic equilibrium there exists particular states of simple system that, macroscopically, are characterized completely by internal energy $\mathrm{U}$, the volume V, and the mole numbers $N_{1}, N_{2}, N_{3}, \ldots \ldots \ldots N_{r}$ of the chemical components.

A physical consequence of symmetry is formulated in "Noethers's theorem". The theorem asserts that every continuous symmetry of the dynamical behavior of a system (i.e. of the dynamical equation and the mechanical potential) implies a conservation law for that system.

The dynamical equation for the motion of the centre of mass point of any material system is Newton's law. If the external force does not depend upon the co-ordinate $\mathrm{x}$, then both the potential and the dynamical equation are symmetric under spatial translation parallel to the $\mathrm{x}$-axis. The quantity that is conserved as a consequence of this symmetry is the $x$-component of the momentum. Similarly the symmetry under translation along the $y$ or $\mathrm{z}$ axes results in the conservation of the $\mathrm{y}$ or $\mathrm{z}$ components of the momentum. Symmetry under rotation around the $\mathrm{z}$-axis implies conservation of the $\mathrm{z}$-component of the angular momentum.

Of enormous significance for thermo-statistics is the symmetry of dynamical laws under time translation. That is, the fundamental dynamical laws of physics (such as Newton's law, Maxwell's equations, and Schrödinger's equation) are unchanged by the transformation $t \rightarrow t^{\prime}+t_{0}$ (i.e., by a shift of the origin of the scale of time). If the external potential is independent of time, Noether's theorem predicts the existence of a conserved quantity. That conserved quantity is called the energy. The evolution of the energy concept for macroscopic thermodynamic systems was even more difficult. The pioneers of the subject were guided neither by a general a priori conservation theorem nor by any specific analytic formula for the energy. Even empiricism was thwarted by the absence of a method of direct measurement of heat transfer. Only inspired in-sight guided by faith in the 
simplicity of nature somehow revealed the interplay of the concepts of energy and entropy, even in the absence of a priori definitions or of a means of measuring either.

\section{The Generalized "First law" of thermodynamics:}

In accepting the existence of a conserved macroscopic energy function as the first postulate of thermodynamics, we anchor that postulate directly in Noether's theorem and the time-translation symmetry of physical laws. There are seven "first integrals of the motion"(as the conserved quantities are known in mechanics). These seven conserved quantities, all arises as Lagrange parameters and they play completely symmetric roles in the theory are the energy, the three components of linear momentums and the three components of the angular momentum; and they follow in parallel fashion from the translation in "space-time" and from rotation. In fact, the energy is not unique in thermo-statistics. The linear momentum and angular momentum play precisely parallel roles. The asymmetry in our account of thermo-statistics is a purely conventional one that obscures the true nature of the subject. The proper "the first law of thermo-dynamics",(or the first postulate in our formulation) is the symmetry of the laws of physics under space-time translation and rotation, and consequent existence of conserved energy, momentum, and angular momentum functions

\section{Goldstone's Theorem with Symmetry Breaking:}

The concept of broken symmetry is clarified by reflecting on the process by which a crystal may be formed. Suppose the crystal to be solid carbon-dioxide ("dry ice"), and suppose the carbon-dioxide initially to be in the gaseous state, contained in some relatively large vessel ("infinite in size"). The gas is slowly cooled. At the temperature of the gas-solid phase transition a crystalline nucleus forms at some point in the gas (which may compared with the very early stages of the universe). The nucleus thereafter grows until the gas pressure falls to that on the gas-solid coexistence curve (i.e., to the vapor pressure of the solid). From the point of view of symmetry the condensation is a quite remarkable development. In the "infinite" gas the system of the universe is symmetric under a continuous translation until the energy group SU(11) of U(11), but the condensed solid (as our universe) has a lower symmetry! It is invariant only under a discrete translation group. Furthermore the location of the crystal is arbitrary, determine by the accident of the first microscopic nucleation. In that nucleation process the symmetry of the system suddenly and spontaneously lowers, and it does so by a nonpredictable, random event. The symmetry of the system is "broken".

Macroscopic sciences, such as solid state physics or thermodynamics, are qualitatively different form "microscopic" sciences because of the effects of broken symmetry, as was pointed out by P.W. Anderson in an early but profound and easily readable essay.

At sufficiently high temperature systems always exhibit the full symmetry of the "mechanical potential" (i.e., of the Lagrangian or Hamiltonian functions). There do exist permissible micro-states with lower symmetry, but these states are grouped in sets which collectively [for example, SU(11)] exhibit the full symmetry. Thus the microstates of a gas do include states with crystal-like spacing of the molecules- in fact, among the microstates all manner of different crystal-like spacing are represented, so that collectively the states of the gas retain no overall crystallinity whatever. However, as the temperature of the gas is lowered the molecules select that particular crystalline spacing of lowest energy, and the gas condenses into the corresponding crystal structure. This is a partial breaking of the symmetry. Even among the microstates with this crystalline periodicity there are a continuum of possibilities available to the system, for the incipient crystal could crystallize with any arbitrary position. Given one possible crystal position there exist infinitely many equally possible positions, slightly displaced by an arbitrary function of a "lattice constant". Among these possibilities, all of equal energy, the system chooses one position (i.e., a nucleation center for the condensing crystallite) arbitrarily and "accidentally". An important general consequence of broken symmetry is formulated in the Goldstone theorem. It asserts that any system with broken symmetry (and with certain weak restrictions on the atomic interactions) has a spectrum of excitations for which the frequency approaches zero as the wave length becomes infinitely large.

So for the crystal discussed here the Goldstone theorem ensures that a phonon excitation spectrum exists, and that its frequency vanishes in the long wave length limit.

In summary, then, the volume emerges as a thermodynamic co-ordinate by virtue of a fundamental symmetry principle grounded in the concept of broken symmetry and in Goldstone's theorem.

\section{Heat Flux in the Early Universe:}

We have discussed that the adiabatic assumption were violated in the stage when the universe was from 10-dimensional to 7-dimensional space-time and then from 7-dimensional to 4-dimensional adiabatic stage arises and then a symmetry breaking of the special unitary group $\mathrm{SU}(11)$, we get mainly two sub-energy groups $\mathrm{SU}(6)$ and $\mathrm{SU}(5)$, where it is possible to change equal number of energy particles (30-number of bosons, which is constant) from the group $\mathrm{SU}(6)$ to $\mathrm{SU}(5)$ or vice-versa and another energy group $\mathrm{U}(1)$. 
We now enquire about the possibility of starting with two arbitrary given state of a system, of enclosing the system in an adiabatic impermeable wall, and of then being able to contrive some mechanical process that will take the system to another arbitrarily specified state. To determine the existence of such processes, we must have recourse to experimental observation, and it is here that the great classical experiments of Joule are relevant. His work can be interpreted as demonstrating that for system enclosed by an adiabatic impermeable wall any two equilibrium states with the same set of mole numbers $N_{1}, N_{2}, \ldots \ldots \ldots, N_{r}$ can be joined by some possible mechanical process. Joule discovered that if two states (say A and B) are specified it may not be possible to find a mechanical process (consistent with an adiabatic impermeable wall) to take the system from A to B but that it is always possible to find either a process to take the system from A to B or a process to take the system from $\mathrm{B}$ to $\mathrm{A}$. this is for any states $\mathrm{A}$ and $\mathrm{B}$ with equal mole numbers, either the adiabatic mechanical process $A \rightarrow \operatorname{Bor} B \rightarrow A$ exists. For out purposes either of these processes is satisfactory. Experiment thus shows that the methods of mechanics permit us to measure the energy difference of any two states with equal mole numbers.

In summary, we have seen that by employing adiabatic walls and by measuring only mechanical work, the energy of any thermodynamic system, relative to an appropriate reference state, can be measured.

The fact that the energy difference of any two equilibrium states is measurable as - The heat flux to a system in any process (at constant mole numbers) is simply the difference in internal energy between the final and initial states, diminished by the work done in that process.

It should be noted that the amount of work associated with different processes initiates in the same state A and each terminates in the same state B. similarly, the heat flux may be different for each of the processes. But the sum of the work and heat fluxes is just the total energy difference $U_{B}-U_{A}$ and is the same for each of the processes. In referring to the total energy flux we therefore need specify only the initial and terminal states, but in referring to heat or work fluxes we must specify in-detail the process considered.

Restricting our attention to thermodynamic simple systems, the quasi-static work is associated with a change in volume and is given quantitatively by

$$
d W_{M}=-P d V \text {, }
$$

Where $\mathrm{P}$ is the pressure. Hence for the quasi-static work, we can now give a quantitative expression for the heat flux. In an infinitesimal quasi-static process at constant mole numbers the quasi-static heat dQ is defined by the equation,

$$
d Q=d U-d W_{M} \quad[\text { at constant mole numbers }] \quad \text { i.e., } \quad d Q=d U+P d V
$$

It will be noted that we use the terms heat and heat-flux interchangeable. Heat, like work, is only a form of energy transfer. Once energy is transferred to a system, either as heat or as work, it is indistinguishable from energy that might have been transferred differently. Thus, although $d Q$ and $d W_{M}$ add together to give dU, the energy U of a state cannot be considered, as the sum of "work" and "heat" components. To avoid this implication we put a strokes through the symbol d: infinitesimals such as $d W_{M}$ and dQ are called imperfect differentials. The integrals of $d W_{M}$ and dQ for a particular process are the work and heat fluxes in that process; the sum is the energy difference $\Delta \mathrm{U}$, which alone is independent of the process.

The whole system of the very early universe may be compared with the concepts of heat, work, and energy which may possibly be clarified in-terms of a simple analogy. A certain farmer owns a pond, fed by one stream and drained by another. The pond also receives water from an occasional rainfall and loses it by evaporation, which we shall consider as "negative rain". In this analogy the pond is our system, the water within it is the internal energy, water transferred by the streams is work, and water transferred as rain is heat.

The first thing to be noted is that no examination of the pond at any time can indicate how much of the water within it came by way of the stream and how much came by way of rain. The term rain refers only to a method of water transfer.

Let us suppose that the owner of the pond wishes to measure the amount of water in the pond. He can purchase flow meters to be inserted in the streams, and with these flow meters he can measure the amount of stream water entering and leaving the pond. But he cannot purchase rain meter. However, he can throw a tarpaulin over the pond, enclosing the pond in a wall impermeable to rain (an adiabatic wall). The pond owner consequently puts a vertical pole into the pond, covers the pond with his tarpaulin, and inserts his flow meters into the streams. By damming one stream and then the other, he varies the level in the pond at will, and by consulting his flow meters he is able to calibrate the pond level, as read on his vertical stick, with total water content $(\mathrm{U})$. Thus, by carrying out processes on the system enclosed by an adiabatic wall, he is able to measure the total water content of any state of his pond.

Our, obliging pond owner now removes his tarpaulin to permit rain as well as stream water to enter and leave the pond. He is then asked to evaluate the amount of rain entering his pond during a particular day. He proceeds simply: he reads the difference in water content from his vertical stick, and from this he deducts the total flux of stream water as registered by his flow meters. The difference is a quantitative measure of the rain. The strict analogy of each of these procedures with its thermo-dynamic counterpart is evident. 


\section{Concluding Remarks:}

We study with quantum black-hole. We discussed three stages of a black-hole. In the first stage, when the universe was from 10-dimensional super-gravity with flat space-time up-to 7-dimensional and then from 7dimensional to 4-dimensional closed black-hole stage, after then 4-dimensional Einstein universe. In my paper it is considered that a symmetry breaking occurred from 7-dimensional universe. We consider a new type of Gaussian energy group SU(6) called latent group and take a single frame-work of the super unified theory, $\mathrm{SU}(11)$, a special unitary group of $11 \times 11$, Hermitian matrices of zero trace, whose sub-sets are as follows $S U(11) \supset S U(6) \times S U(5) \times U(1)$, where $S U(5) \supset S U(3) \times S U(2) \times U(1)$, all energy groups $\mathrm{SU}(3), \mathrm{SU}(2)$ $\& U(1)$ are as usual which was explained in this paper. It was seen from the equations (81) and (86) that the Schwarzschild mass ' $\mathrm{M}$ ' and the angular momentum ' $\mathrm{J}$ ' of the black-hole depends upon the numbers of charge particles ' $\mathrm{e}$ '. We consider the energy group $\mathrm{U}(1)$, are responsible for making the electric charge ' $\mathrm{e}$ ' and hence for charged black-hole. The energy group SU(6) are responsible for strong gravitation field within the blackhole. Also it was shown that 30-number of bosons of SU(6) are changed into 30-number of bosons of SU(5) and vice-versa of the SUT, hence our matter universe (Einstein universe) is emerged from so called black-hole (primordial black-hole) and then everything and also will be destroyed within the black-hole after a long journey.

Every systematic part of the matter universe (i.e., matter energy) controlled by the energy group SU(6) of the black-hole (i.e., latent energy) which is remaining at the centre region primarily. Addition of the part of the matter universe is controlled by the combined black-hole energy. An analogy will illustrate the scenarios of the formation of the so-called black-holes as like as the formation of rain drops from the collecting water vapor from the mixed air in the earth. The black-hole formed in the so-called vapor phase of energy was explained before.

Again, it was shown by considering the line element of the Kantowski-sachs universe, the outer surface $r_{+}$corresponds to the event horizon i.e. $r_{+} \rightarrow M$ as $e \rightarrow 0$. This surface has global significance. The inner surface $r_{-}$is another type of horizon inside the hole itself.

We found that in the flatness problem we lead to fine tuning while for the horizon problem it gave an extremely small size of homogeneity. It therefore appears that the trouble of black-hole lies between10-dimensional to 7dimensional flat universe and could be resolved as the adiabatic assumption were violated at this stage. As we consider the universe started from 10-dimensional space-time, so the origin of time shifted at $t \rightarrow t_{0}$. We showed the conservation principle of energy, by considering the fiducial state of energy as SU(11) is arbitrarily taken as zero.

It is shown that the conservation law of energy is maintained by introducing internal energy at the beginning of our universe by considering the fiducial state of system, where the energy of which is arbitrarily taken as zero, and hence in the C-field, it is possible to increase the matter-mass of our 4-dimensional universe. The energy difference of any two equilibrium states is measurable as: The heat-flux to a system in any process (at constant mole numbers) is simply the difference in internal energy between the final and initial states, diminished by the work done in that process.

\section{Acknowledgement:}

The author is thankful to Prof. Subenoy Chakraborty, Department of Mathematics, Jadavpur University, Kolkata, West Bengal, India, for his helpful discussions.

\section{References:}

[1]. Hawking S.W(1984): The quantum state of the universe, Nucl. Phys. B239.257.

[2]. Hoyle F. and Naralikar J.V(1964): A new theory of gravitation. Proc. R. Soc., A282.191.

[3]. Bhadra N.K(2012):The complex Model of the Universe, IOSR-JM, ISSN: 2278-5728, vol.2, 4, pp-20; and The complex model of the quantum universe,vol.4, 1, pp-20.

[4]. Einstein, A. de-Sitter, W. (1932): On the relation between the expansion and mean density of the universe. Proc. Natl. Acad. Sci.,(USA), 18,213.

[5]. Davies, P.C.W. (1974): The Physics of Time Asymmetry(Surrey University Press/ University of California Press) - 1976a Proc. R. Soc. A. 351 139; -1976b Nature 263 377; -1977a Proc. R. Soc. A. 353 499; -1977b Space and Time in the Modern University (Cambridge : Cambridge University Press).

[6]. Mendeez, V. and Pavon, D.(1996): Gen. Rel. Grav., 28. 697; Birrell N. D and Davies, P.C.W 1978 Nature 27235

[7]. Bekenstein, J.D.1973 Phys. Rev. D. 72333; Bertin, G., and Radicati, L.A 1976 Astrophys.206 825;.

[8]. Candelas P., and Seiama, D.W. 1977 Phys. Rev. Lett. 38 1372; Carr, B. 1977 Mon, Not. R. Astrom. Soc. 181 293; Carter B 1973 Black-Holes ed DeWitt and DeWitt (London: Gordon and Breach) Casimir HBG 1948 Proc. Kon. Ned. Akad. Wetens chap. 51793.

[9]. Christensen, S.M. and Fulling, S.A. 1977 Phys. Rev. D15 2088;Einstein Albert (1987): Ideas and Opinions, Crown Publishers, New York, pp-348.

[10]. Halliwel, J.J. Hawking, S.W., (1985): The Origin of Structure in the Universe, Phys. Rev. D 31 1777. Hawking, S.W.(1985): The Arrow of Time in Cosmology Phys. Rev., D 32 2489. 1.

[11]. Hawking, S.W. and Ellis GFR 1973, The Large Scale Structure of Space-Time (Cambridge: Cambridge University Press).

[12]. Guth, Alan. H.(1997): The inflationary Universe, Addision-Wesley Co., Inc. Reading, Massachusetts. 
[13]. Guth, A. and Steinhardt. Paul(1992): The inflationary Universe and article in The New Physics edited by Paul Davies (Cambridge university Press). Pp-55. Smarr L 1973 Phys. Rev. Lett. 3071.

[14]. Appelquist, T., Chodos, A. and Freund, P.G.O (1986): Modern Kaluza-Klein Theories, Frontiers in Physics in series Vol. 65, (Ed. Adison-Wesley). A.G. Riess et al, Astrophys ., 560,49(2001).

[15]. Abdul Rahaman, A.M.M.(1992): Phys.Rev. D 45, 3497; Abbott, L.F. (1985): Phys. Lett. 150B, 427. Giddings, S.B. and Strominer, A. (1988-89): Nucl. Phys., B 307, 854 B 321, 481(1989).

[16]. Emmy Noether, Gesammette Abhandlungen, (Collected Papers), Springer-Verlag, Berlin-New-York, 1983.

[17]. Wigner, E. 'Symmetry and Conservation Laws', Physics Today, March 1964 P.34. Anderson, P.W., pp 175-182 in concepts in solids (W.A. Benjamin Inc., New-York, 1964).

[18]. Parratt, L.G., Probability and Experimental Errors in Science, Wiley, New-York, 1961 and Parzen, E., Modern Probability Theory and Its Application, Wiley, New-York, 1960.

[19]. C. Huang, Statistical Mechanics, Wiley, 1963. Classic graduate text. ; R.J.Finkelstein, Thermodynamics and Statistical Physics-A Short Introduction, W. H. Freeman and Co., 1969.

[20]. M. Tribus, Thermostatics and Thermodynamics, Van Nostrand, 1961. ; P. T. Landsburg, Thermodynamics and Statistical Mechanics, Oxford University Press, 1978. ;

[21]. P.W. Anderson, Basic Notions of Condensed Matter Physics, Benjamin/Cummings, 1984. ; J. W. Gibbs, The Scientific Papers of J. Willard Gibbs, Volume I, Thermodynamics, Dover, 1961.

[22]. Herbert.B. Callen, Books, General Principles of Classical Thermodynamics. Philadelphia, Pennsylvania. 